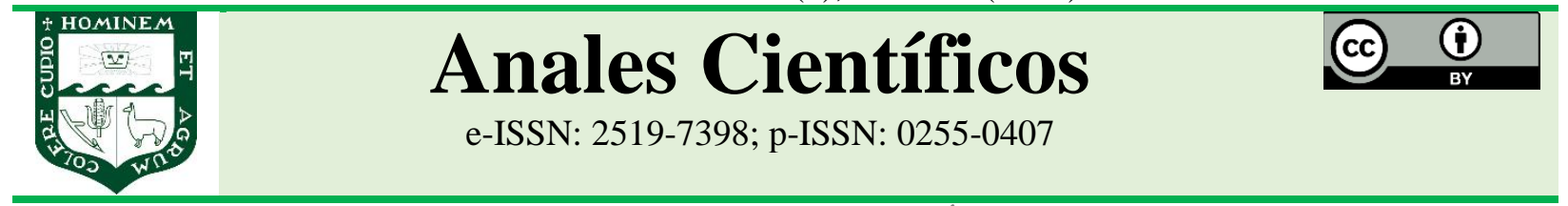

ARTÍCULO ORIGINAL - RESEARCH ARTICLE

http://dx.doi.org/10.21704/ac.v82i2.1790

\title{
RIESGO EN LA AGRICULTURA: EL CASO DE LA PAPA (Solanum tuberosum) EN AYACUCHO Y LIMA
}

\section{Risk in agriculture: the case of the potato (Solanum tuberosum) in Ayacucho and Lima}

\author{
Raquel Gómez Oscorima ${ }^{1 *}$; ; Ramón Diez Matallana ${ }^{1}$ (D); María Anderson-Seminario² (D); \\ Pether López García ${ }^{1}$
${ }^{1}$ Facultad de Economía y Planificación, Universidad Nacional Agraria La Molina, La Molina 15024, Perú.
${ }^{2}$ Universidad de Lima, Santiago de Surco 15023, Perú.
*E-mail: rgo@lamolina.edu.pe.

Recibido: 04/08/2020; Aceptado: 02/10/2021 Publicado: 31/12/2021

\begin{abstract}
Agricultural production is very risky, especially in the case of potatoes, and the risk is differentiated between the Coast and the Sierra. On the coast, Lima shows high yields per hectare while, in the Sierra, Ayacucho shows low yields. In order to compare the risk levels in potato producers in the Ayacucho and Lima regions, and the incidence of probabilistic variables (farm price, yield per hectare, costs of fertilizers, seeds, pesticides) in the margin gross economic benefit per hectare, a stochastic Monte Carlo simulation is carried out with @Risk software. The hypothesis that Ayacucho would present higher levels of risk than Lima was tested. It was found that Lima has $65.5 \%$ of positive gross margin scenarios and Ayacucho only 1.6\%. The main sources of risk in white potato production are the price on the farm and the yields per hectare. The variation of the gross margin per hectare due to increases in the farm price of $10 \%$ is $6.6 \%$ for Ayacucho and $9.1 \%$ for Lima. In the case of yields, a $10 \%$ increase in yield raises the margin by $4.6 \%$ in Ayacucho, while in Lima, only 3.3\%. It is concluded that yields in Ayacucho should be increased to improve profits.
\end{abstract}

Keywords. Potato | random variable | risk | stochastic simulation of Montecarlo.

\section{RESUMEN}

La producción agrícola es muy riesgosa, especialmente en el caso de la papa, y el riesgo es diferenciado entre la Costa y la Sierra. En la Costa, Lima muestra altos rendimientos por hectárea mientras que, en la Sierra, Ayacucho muestra bajos rendimientos. Con el objetivo de comparar los niveles de riesgo en los productores de papa blanca en las regiones de Ayacucho y Lima, y la incidencia de las variables probabilísticas (precio en chacra, rendimiento por hectárea, costos de fertilizantes, semillas, pesticidas) en el margen bruto de beneficio económico por hectárea, se efectúa una simulación estocástica de Montecarlo, con el software @Risk. Se sometió a prueba la hipótesis de que Ayacucho presentaría mayores niveles de riesgo que Lima. Se encontró que Lima tiene 65,5\% de escenarios de margen bruto positivo y Ayacucho sólo 1,6\%. Las principales fuentes de riesgo en la producción de papa blanca, son el precio en chacra y los rendimientos por hectárea. La variación del margen bruto por hectárea por incrementos en el precio en chacra de un 10\% es de 6,6\% para Ayacucho y de 9,1\% para Lima. En el caso de los rendimientos 
un incremento de $10 \%$ en rendimiento eleva el margen en 4,6\% en Ayacucho, mientras en Lima, sólo 3,3\%. Se concluye que debe incrementarse los rendimientos en Ayacucho para mejorar los beneficios.

Palabras clave: Papa | riesgo | simulación estocástica de Montecarlo | variable aleatoria.

Forma de citar el artículo (Formato APA):

Gómez, R., Diez, R., Anderson, M., \& López, P. (2021). Riesgo en la agricultura: el caso de la papa (Solanum tuberosum) en Ayacucho y Lima. Anales Científicos. 81(2), 279-287. http://dx.doi.org/10.21704/ac.v82i2.1790

Autor de correspondencia (*) Raquel Margot Gómez Oscorima. Email: rgo@lamolina.edu.pe

(C) Los autores. Publicado por la Universidad Nacional Agraria La Molina.

This is an open access article under the CC BY

\section{INTRODUCCIÓN}

La evaluación de riesgo es esencial para la toma de decisiones en la agricultura, especialmente en papa (Solanum tuberosum), el segundo cultivo por volumen producido y el tercero en superficie cosechada en Perú (Coaquira et al., 2019), especialmente en los departamentos de Puno, Huánuco, La Libertad, Apurímac, Cusco y Junín. Este cultivo involucra 710 mil familias con menos de media hectárea por familia, ocupando 33,4 millones de jornales y contribuyendo con $4 \%$ del PBI agrícola.

Según Coaquira et al. (2019); Bonnet (2018); el mundo considera la papa uno de los alimentos esenciales y es el cuarto más consumido después del maíz, trigo y arroz. En producción, Perú ocupa la duodécima posición en el mundo y el primero en América Latina, pero su rendimiento promedio $(13,25 \mathrm{t} / \mathrm{h})$ es menor que el promedio mundial $(15,85 \mathrm{t} / \mathrm{h})$ y por ello, Anderson et al. (2019) señalan que Perú no es competitivo ni se posiciona como exportador. Respecto a ello, Oliver (2017) señala que Estados Unidos logra 46,4 t/h, Alemania 43,9 t/h, Chile 23,7 t/h y Brasil 18,39 t/h.

Guillén (2019), señala que, en Perú, el rendimiento en papa se reduce por las plagas y enfermedades, y ello acarrea pérdidas económicas para muchos productores. En tanto que, de Almeida et al. (2015); Bonnin et al. (2017), Figueroa et al. (2019), coinciden en que los agricultores logran baja productividad y rentabilidad por el uso limitado de semillas certificadas, sequía y prácticas agrícolas deficientes, lo cual estaría asociado con escasa dotación de capital según Mejía y Castellanos (2018). Por otro lado, Basantes et al. (2020); Aragón et al. (2020), evalúan la producción y comercialización de papa en Ecuador señalan que el $81.80 \%$ del costo de producción de papa se explica por el gasto en insumos y que los productores grandes obtienen rendimientos mayores en más de $30 \%$ que los pequeños productores $\mathrm{y}$, en consecuencia, mayor rentabilidad.

En Perú, la papa se cultiva en 19 de sus 24 regiones, de 100 a 4200 metros sobre el nivel del mar (MINAGRI, 2018); lo cual ya señalaba Scott (1985), quien resaltó su presencia en el portafolio productivo de muchos agricultores. Respecto a mejoras técnicas, Guillén (2019), señala que en Perú podría emplearse papa transformada genéticamente para resistir enfermedades como la rancha o tizón tardío causada por la Phytopthora infestans, y el tizón temprano causado por Alternaria solani, usando material genético de variedades silvestres de papa. En ese sentido, Cunya et al. (2017), resaltan las ventajas por menores costos y resistencia del grupo Phureja a rancha causada por Phytophthora infestans, amarillamiento de venas causada por un virus, marchitez bacteriana por Ralstonia solanacearum, sarna polvosa por Spongospora subterránea; y a la polilla guatemalteca (Tecia solanivora Povolny). Por estas ventajas el grupo Phureja podría aportar genes valiosos para la mejora genética de la papa, sea convencional o por recombinación de ADN.

Los productores de papa son pobres y de bajo nivel de ingresos; por lo que es necesario analizar la incidencia de los factores productivos en la rentabilidad en Ayacucho y Lima. La rentabilidad de los productores ayacuchanos es menor, y está asociada con altos niveles de riesgo, amenazando su subsistencia (López, 2019). Un trabajo que compara la influencia de los determinantes en la rentabilidad en papa de Huánuco y Lima realizado por Minaya (2015), usó el análisis de simulación de Montecarlo y encontró que la principal fuente de riesgo es el precio en chacra de papa. López 
(2019), comparó el riesgo en la producción de papa en Ayacucho y Lima, encontrando que es mayor en Ayacucho.

Respecto al uso de distribuciones de probabilidad para evaluar rentabilidad bajo riesgo, López (2019) cita a Lobos et al. (2015), quienes aplican distribuciones de probabilidad para las variables de entrada (SIV), que simularon y validaron. Con los valores aleatorios de SIV obtuvieron valores aleatorios de producción, ingresos, costos, depreciación, impuestos y flujos de efectivo netos y estimaron las distribuciones de probabilidad de las variables de salida estocásticas (SOV): valor presente neto, tasa interna de rendimiento, valor en riesgo, costo promedio de producción, margen de contribución y rendimiento del capital. Cuantificado el riesgo, es decir, determinados los posibles resultados y su probabilidad de ocurrencia, se usa distribuciones de probabilidad para describir los escenarios posibles.

La inversión en papa presenta rentabilidad diferenciada según la región y ello nos lleva a preguntarnos: ¿Es menor la rentabilidad en Ayacucho porque la mano de obra y los insumos y servicios de maquinaria agrícola son más caros que en Lima o es sólo un problema de bajos rendimientos por hectárea? ¿O nos alineamos con Minaya (2019), para quien son los mayores precios de papa en Lima, respecto a los de otras localidades, los que explican las diferencias en la rentabilidad?

Así, el objetivo es comparar la rentabilidad económica de los productores de papa blanca en Lima y Ayacucho en condiciones de riesgo y comparar la incidencia de los factores explicativos en ambas localidades.

\section{MATERIALES Y MÉTODOS}

Hipótesis: Los precios en chacra y los rendimientos por hectárea de papa blanca determinarían los niveles de rentabilidad y los niveles de riesgo; por ello, la rentabilidad económica de la región de Lima es más alta y con menor riesgo que la región de Ayacucho.

\section{Las regiones y su aporte al suministro de papa para Lima}

Región Ayacucho. Según López (2019), se ubica en la zona centro-sur de los Andes peruanos, su área: 43,8 mil $\mathrm{Km}^{2}, 3,4 \%$ del territorio nacional, 616 mil habitantes. Se siembra papa en más de 23 mil hectáreas en 2018 y es el octavo proveedor de papa en Perú, y produce más de 425 mil toneladas ( $8 \%$ de la producción nacional).

Región Lima. Se ubica en la costa central del país, adyacente al Océano Pacífico, la extensión de Lima es de 34,9 mil $\mathrm{km}^{2}, 2,7 \%$ del territorio nacional. La población de Lima es de 9,48 millones de habitantes. La agricultura es la mayor fuente de empleo en Lima. En 2018, Lima produjo 122 mil toneladas de papa, 2,38\% de la producción total del país, con un rendimiento de 23,35 toneladas/hectárea en 2018 frente a 17,69 toneladas/hectárea en Ayacucho y 15,85 toneladas/hectárea en Perú (López, 2019).

Importancia de la papa blanca de Lima y Ayacucho en el mercado mayorista de Lima. Las papas producidas en Lima y vendidas en el Mercado No.1 constituyen el $17,55 \%$ de la participación total, y Ayacucho aporta un 5\% (López, 2019).

\section{Metodología}

A partir del presupuesto por hectárea de producción de papa en Ayacucho y Lima, se simula ingresos por ventas, considerando valores esperados de rendimientos y precios en chacra. Para obtener el margen bruto de beneficio por hectárea, se resta del ingreso los costos totales.

Las variables de riesgo, llamadas variables de entrada o probabilísticas son: precios, rendimientos, fertilizantes, pesticidas, semillas, maquinaria, que generan múltiples escenarios usando la simulación de Montecarlo (Figueroa et al., 2019). La simulación brinda resultados probabilísticos y gráficos, sensibilidad y análisis de escenarios y correlación de variables de entrada. A partir de escenarios generados por las variables de entrada se obtienen distribuciones de probabilidad de la variable de salida: margen bruto de rentabilidad por hectárea. En la Tabla 1 se ve las variables de entrada y salida.

Distribuciones de probabilidad utilizadas: Seguimos a López (2019), que emplea las distribuciones: 1) Uniforme, donde los posibles resultados entre un mínimo y un máximo son igualmente probables. 2) Normal: La densidad más alta se acerca al promedio y se caracteriza por dos parámetros: la media, $\mu$ (parámetro de ubicación) y la desviación standard $\sigma$ (parámetro de escala). 3) Triangular: Definida por los 
parámetros: mínimo (a), valor más probable (b) y el máximo (c).

Tabla 1. Variables de entrada y salida.

\begin{tabular}{ll}
\hline Variables de salida & Variables de entrada \\
\hline & Precio en chacra de papa en \\
& Soles/Kg \\
Indicador de & Rendimiento en \\
rentabilidad: & Toneladas/hectárea \\
Margen Bruto por & Costo de fertilizantes en \\
hectárea (beneficios & Soles/hectárea \\
económicos). & Costo de semillas en \\
& Soles/hectárea \\
& Costo de pesticidas en \\
& Soles/hectárea \\
& Costo de mano de obra en \\
& Soles/hectárea \\
& Costo de maquinaria en \\
& Soles/hectárea \\
\hline
\end{tabular}

Fuente: Minaya (2015)

Variables en estudio. Las variables son de dos tipos: determinísticas y probabilísticas. Las determinísticas con probabilidad de ocurrencia de $100 \%$ (tienen desviación estándar baja con respecto a su valor promedio). Las probabilísticas tienen niveles significativos de variabilidad de una campaña a otra, con alta desviación estándar respecto a su promedio.

Ingresos. Los ingresos (soles por hectárea) de los productores de papa resultan de dos variables probabilísticas en el modelo: precios en chacra (soles por kilo) y rendimientos por hectárea durante la campaña agrícola (kilos). Presentan un grado alto de variabilidad de campaña a campaña, por factores de mercado, ambientales y la gestión de cultivos. Por esta razón, los precios y rendimientos son variables probabilísticas o asociadas al riesgo.

\section{Descripción de las variables de entrada (probabilísticas)}

Precio en chacra: Por las variaciones del mercado y el variado poder de negociación de los agricultores, se ajusta a una distribución de probabilidad uniforme en Ayacucho y a una triangular en Lima.

Rendimiento por hectárea: Alta variabilidad, depende de factores ambientales y del manejo agronómico. Distribución de probabilidad uniforme.

Costos de producción: Según López (2019) son gastos por fertilizantes, semillas, pesticidas, mano de obra, mecanización y costos indirectos. Hay probabilísticos y determinísticos.

c.1. Costos probabilísticos: Para López (2019) son gastos en Fertilizantes (Nitrato de amonio, Fosfato diamónico, Cloruro de potasio), Semilla (por alta participación en costos totales), Plaguicidas (Ciflutina, Carbofuran, Carboxin, Mancozeb, Propineb y Cymoxanil), fuerza de trabajo (alta variabilidad de salarios) y Maquinaria (alta variabilidad según la zona). c.2. Costos determinísticos: Agua y otros, relativamente constantes y de pequeña dimensión en las campañas de producción.

\section{Comparación de los costos de producción de papa blanca}

La estructura de costos de producción de papa para 2015 se ve en la Tabla 2, la cual muestra valores esperados de los diversos costos de producción de los años 2007 a 2015, mostrados en el Anexo 1.

Tabla 2. Costo total de producción de papa por hectárea 2015.

\begin{tabular}{|c|c|c|c|c|}
\hline Ítems & Ayacucho S/. & Lima S/. & Ayacucho \% & Lima \% \\
\hline Fertilizantes & 2887,90 & 3967,40 & $25,9 \%$ & $28,9 \%$ \\
\hline Semilla & 1876,40 & 2850,00 & $16,8 \%$ & $20,8 \%$ \\
\hline Pesticidas & 1400,50 & 1400,20 & $12,6 \%$ & $10,2 \%$ \\
\hline Mano de obra & 3007,50 & 2987,60 & $27,0 \%$ & $21,8 \%$ \\
\hline Maquinaria & 1078,50 & 1112,30 & $9,7 \%$ & $8,1 \%$ \\
\hline Otros costos & 890,40 & 1375,60 & $8,0 \%$ & $10,0 \%$ \\
\hline Costo Total & 11141,20 & 13693,10 & $100,0 \%$ & $100,0 \%$ \\
\hline
\end{tabular}


En ambas regiones, se usa tecnología media y el agricultor es el propietario de la tierra, por eso no se considera alquiler de la tierra.

\section{La Simulación de Montecarlo}

La rentabilidad por hectárea (margen bruto), en un entorno de riesgo, en la producción de papa blanca es representada por la letra Y, según la Fórmula 1.

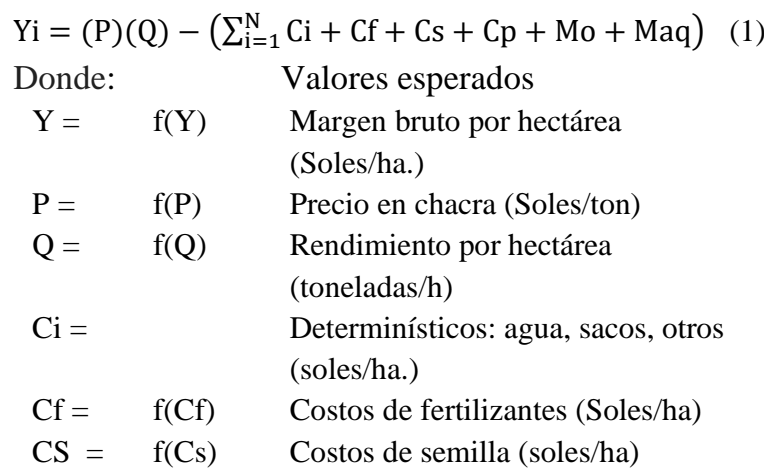

$$
\begin{aligned}
& \mathrm{Cp}=\mathrm{f}(\mathrm{Cp}) \quad \text { Costos de pesticidas (soles/ha) } \\
& \mathrm{Mo}=\mathrm{f}(\mathrm{Mo}) \quad \text { Costos de mano de obra (soles/ha) } \\
& \mathrm{Maq}=\mathrm{f}(\mathrm{Maq}) \quad \text { Costos de maquinaria (soles/ha.) } \\
& \text { i = Región Ayacucho, Región Lima. }
\end{aligned}
$$

Los costos más significativos de producción (más de $70 \%$ del total) de papa blanca en Ayacucho y Lima corresponden a mano de obra, fertilizantes y semilla. Los costos determinísticos son de 8 a $10 \%$ de los costos totales.

En la Tabla 3 se muestra los parámetros de distribución de cada variable; mínimo, máximo, para distribuciones uniformes, el promedio y la desviación estándar corresponden a distribuciones normales, para la distribución triangular son el mínimo, máximo y la moda. Así, para los precios corresponde la distribución Triangular. Los parámetros determinan la probabilidad de ocurrencia de diferentes escenarios.

Tabla 3. Distribuciones de probabilidad de las variables de entrada.

\begin{tabular}{lccccc}
\hline \multirow{2}{*}{ Ítem } & \multirow{2}{*}{ Unidades } & \multicolumn{2}{c}{ Ayacucho } & Lima \\
\cline { 3 - 6 } & & Distribución & Parámetros & Distribución & Parámetros \\
\hline Precio en chacra & $\mathrm{S} / \mathrm{Kg}$ & Uniforme & 0,$29 ; 0,67$ & Triangular & 0,$31 ; 0,47 ; 1,08$ \\
Rendimiento & $\mathrm{Kg} / \mathrm{ha}$ & Uniforme & $9448 ; 16700$ & Uniforme & $18116 ; 25605$ \\
Fertilizantes & $\mathrm{S} / \mathrm{ha}$ & Uniforme & 2135,$29 ; 3890$ & Uniforme & $1890 ; 4224$ \\
Semilla & $\mathrm{S} / \mathrm{ha}$ & Uniforme & $960 ; 2250$ & Triangular & $1750 ; 3000 ; 3000$ \\
Pesticidas & $\mathrm{S} / \mathrm{ha}$ & Uniforme & $925 ; 2711$ & Normal & $830 ; 1400,2$ \\
Mano obra & $\mathrm{S} / \mathrm{ha}$ & Uniforme & 1424,$18 ; 4585$ & Uniforme & $1575 ; 2987,6$ \\
Maquinaria & $\mathrm{S} / . / \mathrm{ha}$ & Uniforme & 1078,$5 ; 1131,43$ & Triangular & $745 ; 1237,5 ; 1237.5$ \\
\hline
\end{tabular}

\section{RESULTADOS Y DISCUSIÓN}

Se muestra las distribuciones de probabilidad del margen bruto en cada región en la Tabla 4. Asimismo, se usa el análisis de sensibilidad para medir los efectos de las variables más críticas en los niveles de beneficio económico por hectárea en la producción de papa blanca.

\section{Beneficios por hectárea en papa en Ayacucho y Lima}

Los estadísticos del margen bruto (indicador de beneficios) se obtuvieron con diez mil iteraciones. La diferencia entre las regiones es notoria, Lima obtiene una rentabilidad promedio de 2145,2 soles y en Ayacucho hay una pérdida esperada de $-4802,8$ soles. El valor más frecuente (moda) en Ayacucho es -
5004,90 soles mientras en Lima es de 1172,20 soles. En cuanto al máximo, en Ayacucho es 2122 mientras que en Lima llega a 15800. Los menores ingresos en Ayacucho estarían reflejando el menor nivel tecnológico y la difícil geografía de la región.

\section{Resultados del modelo probabilístico}

En la figura 1 se observa que el margen bruto por hectárea de la producción de papa blanca en Ayacucho tiene un mínimo de - 10964,50 soles (pérdidas) y un beneficio máximo de 2122,70 soles, con un promedio de $-4802,80$ soles.

En Ayacucho, los agricultores suelen obtener pérdidas económicas, ya que más del $95 \%$ de los escenarios presenta resultados negativos, y menos del 5\% presenta niveles de beneficios económicos del orden 
de 2200 soles, considerando las probabilidades de distribución de precios y rendimientos por hectárea, y los costos de mano de obra, maquinaria, fertilizantes, semillas, y otros, se encuentra que el agricultor papero de Ayacucho tiene más probabilidades de tener pérdidas económicas.

Tabla 4. Estadísticos del Margen Bruto por hectárea.

\begin{tabular}{|c|c|c|}
\hline Estadístico & Ayacucho & Lima \\
\hline Número de iteraciones & $\overline{10000,00}$ & 10000,00 \\
\hline Promedio & $-4802,80$ & 2145,20 \\
\hline Desviación standard & 2191,40 & 4018,60 \\
\hline Mediana & $-4912,90$ & 1581,90 \\
\hline Moda & $-5004,90$ & 1172,20 \\
\hline Mínimo & $-10964,50$ & $-6911,40$ \\
\hline Máximo & 2122,80 & 15800,80 \\
\hline Coeficiente de variabilidad & 0,46 & 1,88 \\
\hline
\end{tabular}

El análisis probabilístico refleja una gran dispersión de resultados en cada región. Explicable, por la variabilidad de las condiciones de producción de los agricultores, ligadas a su diverso tamaño, clima (especialmente en Ayacucho), nivel de tecnología y grado de capitalización.

En la Figura 2, los beneficios económicos (margen bruto por hectárea) de la papa blanca en Lima varían desde un mínimo de - 6911,40 soles a un máximo de 15800 soles, con un promedio esperado de 2145,20 soles.

En la Figura 3 se aprecia que, en Lima, los paperos tienen $65,5 \%$ de probabilidades de tener beneficios mientras que en Ayacucho sólo tienen 1,6\%.

\section{Análisis de sensibilidad}

La Tabla 5 relaciona las medidas de riesgo, es decir, la probabilidad de obtener ciertos niveles de rentabilidad con cada uno de los 20 rangos de probabilidad del 0 al $100 \%$, en clases del $5 \%$.

La Tabla 6 muestra los coeficientes de sensibilidad del margen bruto para las 2 regiones, se puede ver los impactos diferenciados de cada variable en Ayacucho y en Lima. Se muestra la interpretación de los coeficientes de sensibilidad.
El precio en chacra tiene un impacto relativo más fuerte en Ayacucho (0.914) que en Lima (0.663). En cambio, el rendimiento es un poco más impactante en Lima (4.69) que en Ayacucho (3.30).

En Ayacucho, cuando el precio en chacra de la papa aumenta en 10 por ciento, el margen bruto por hectárea aumenta en 9,14 por ciento. Si el rendimiento por hectárea aumenta un diez por ciento, el margen bruto aumenta sólo un 3,36 por ciento, menor impacto que en Lima.

En cuanto a los costos, los impactos de todos son importantes, reduciendo proporcionalmente más el margen bruto en Lima que en Ayacucho. Aumentos en el costo de los pesticidas en un 10 por ciento disminuirán el margen bruto en un 0,43 por ciento. Cuando el costo de las semillas aumenta en 10 por ciento, el margen bruto por hectárea disminuirá en un 0,74 por ciento. En general, los incrementos de costos en Lima, reducirían más el margen bruto que en Ayacucho. Es decir, si los precios en chacra son mayores en Lima, también lo son los costos.

Lima es más competitiva por rentabilidad en papa, y con menores niveles de riesgo. Los beneficios económicos en Lima son mayores que los de Ayacucho. En casi todos los escenarios $(98,4 \%)$ se obtiene pérdidas en Ayacucho, mientras que en Lima se obtiene ganancias en $65,5 \%$ de los escenarios.

Las principales fuentes de riesgo para ambas regiones son los precios en chacra de papa, y el rendimiento por hectárea y, en menor medida, los costos de fertilizantes y fuerza laboral.

El precio en chacra, en ambas regiones es la variable de mayor riesgo para los agricultores, y sus niveles de impacto son del $66.3 \%$ para Ayacucho y del $91.4 \%$ para Lima, mientras que el rendimiento por hectárea, en Ayacucho, representa un impacto del 46\%, y en Lima un impacto del 33\%. Cabe señalar que, los resultados son análogos a los obtenidos por Minaya (2015) en su comparación entre Huánuco y Lima. 


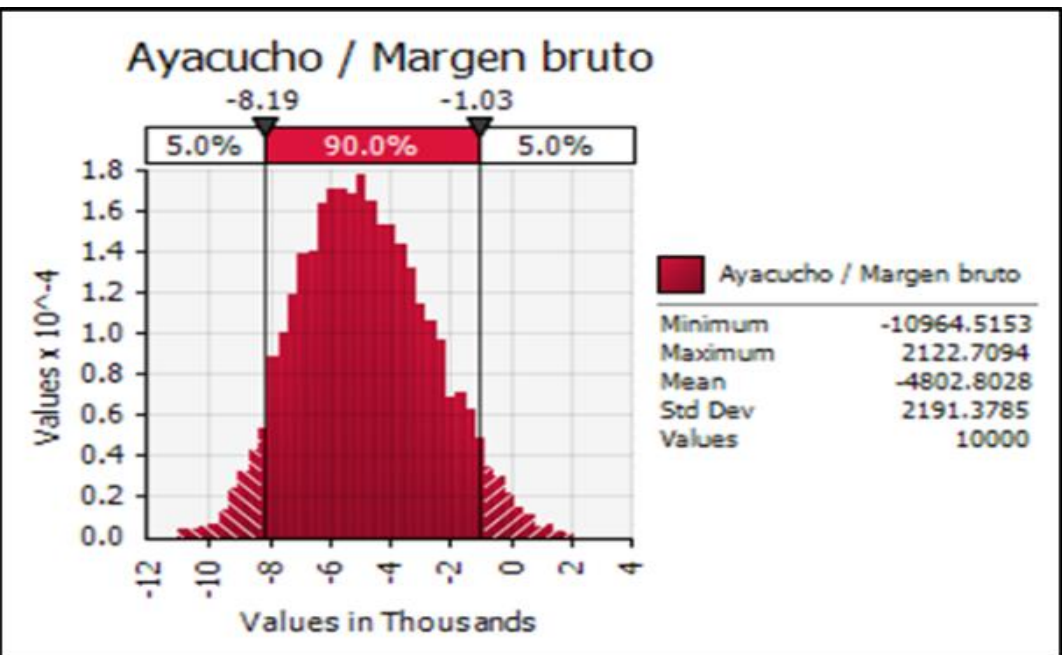

Figura 1. Distribución de probabilidad del margen bruto (indicador de beneficios económicos) en Ayacucho.

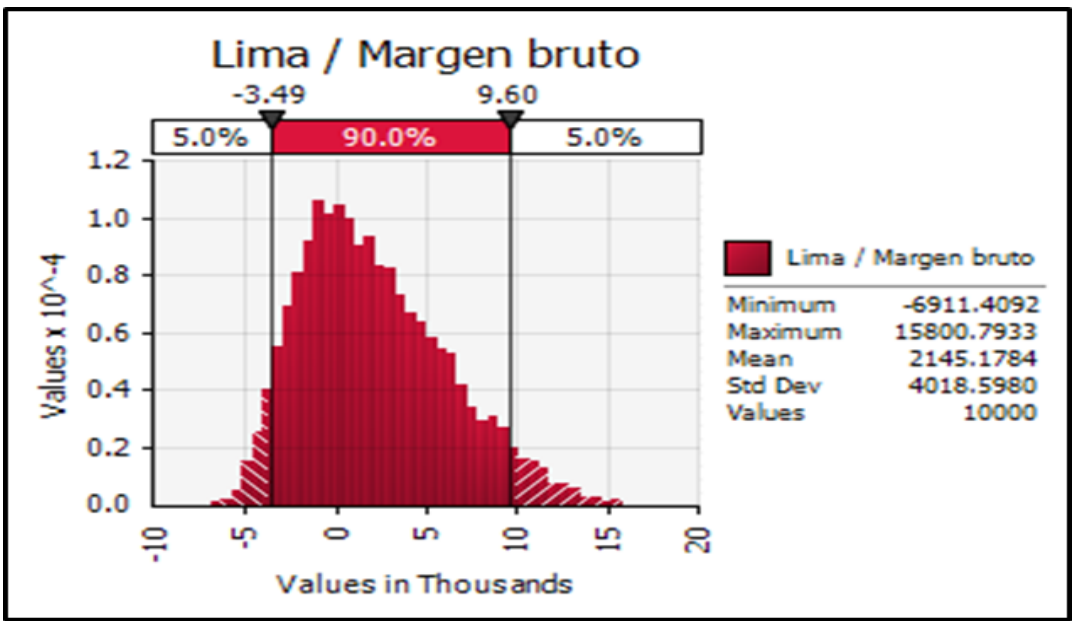

Figura 2. Distribución de Probabilidad del margen bruto (indicador de beneficios económicos) en Lima.

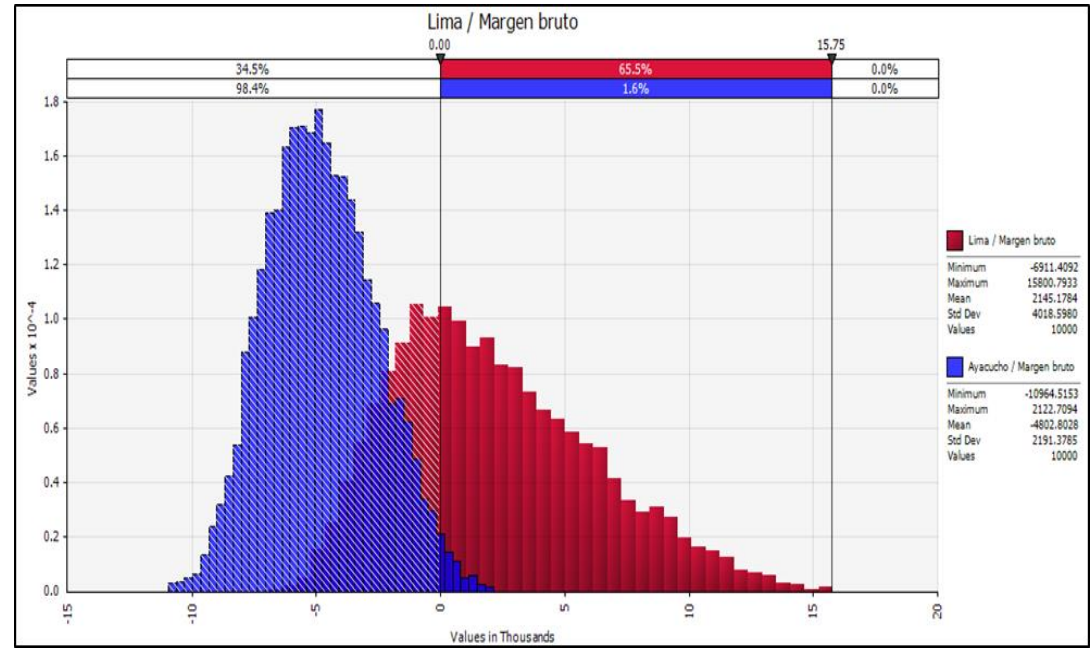

Figura 3. Distribución de probabilidad de margen bruto por hectárea para Lima (rojo) y Ayacucho (azul). 
Tabla 5. Percentiles de riesgo del margen bruto por hectárea.

\begin{tabular}{ccc}
\hline $\begin{array}{c}\text { Nivel de } \\
\text { Riesgo } \\
\text { Región }\end{array}$ & $\begin{array}{c}\text { Margen } \\
\text { Bruto/ha } \\
\text { Ayacucho }\end{array}$ & $\begin{array}{c}\text { Margen } \\
\text { Bruto/ha } \\
\text { Lima }\end{array}$ \\
\hline $0 \%$ & $-10964,52$ & $-6911,41$ \\
$5 \%$ & $-8188,23$ & $-3490,88$ \\
$10 \%$ & $-7575,85$ & $-2652,27$ \\
$15 \%$ & $-7117,22$ & $-1977,42$ \\
$20 \%$ & $-6747,01$ & $-1418,63$ \\
$25 \%$ & $-6391,93$ & $-943,85$ \\
$30 \%$ & $-6086,82$ & $-449,02$ \\
$35 \%$ & $-5795,48$ & 49,18 \\
$40 \%$ & $-5503,71$ & 521,10 \\
$45 \%$ & $-5202,87$ & 1031,11 \\
$50 \%$ & $-4912,87$ & 1581,95 \\
$55 \%$ & $-4624,01$ & 2131,02 \\
$60 \%$ & $-4317,86$ & 2726,60 \\
$65 \%$ & $-3992,96$ & 3334,54 \\
$70 \%$ & $-3652,68$ & 4040,11 \\
$75 \%$ & $-3296,37$ & 4807,54 \\
$80 \%$ & $-2891,32$ & 5643,26 \\
$85 \%$ & $-2417,61$ & 6561,57 \\
$90 \%$ & $-1805,75$ & 7860,23 \\
$95 \%$ & 1029,26 & 9602,42 \\
$100 \%$ & 2122,71 & 15800,79 \\
\hline
\end{tabular}

\section{CONCLUSIONES}

Los productores de Lima tienen mayor rentabilidad esperada promedio por hectárea $(2145,17)$ que los de Ayacucho $(-4802,80)$ y menores niveles de riesgo pues Lima tiene $34,5 \%$ de escenarios negativos y Ayacucho $98,4 \%$. El mayor rendimiento por hectárea de los agricultores de Lima explica la mayor rentabilidad, que se confirma por el coeficiente de sensibilidad $(0,469)$ mayor al de Ayacucho (0,330). El precio en chacra tiene mucha influencia en los niveles de rentabilidad, pero en Ayacucho no alcanza a revertir los efectos del bajo rendimiento por hectárea. Se sugiere realizar nuevos trabajos comparativos sobre la rentabilidad en productores de papa que adoptan tecnologías alternativas de producción como el control integrado de plagas versus agricultura convencional o comparar el uso de diversas semillas como hizo Guillén (2019).

Tabla 6. Coeficientes de sensibilidad del margen bruto y su interpretación.

\begin{tabular}{lcccc}
\hline \multicolumn{1}{c}{ Región } & Lima & Ayacucho \\
\hline $\begin{array}{c}\text { Variable } \\
\text { explicativa } \\
\text { (VE) }\end{array}$ & $\begin{array}{c}\text { Coeficiente } \\
\text { de Sensibilidad }\end{array}$ & $\begin{array}{c}\text { Interpretación } \\
\text { Si aumenta VE en 10\% entonces } \\
\text { el Margen bruto: }\end{array}$ & $\begin{array}{c}\text { Coeficiente } \\
\text { de } \\
\text { Sensibilidad }\end{array}$ & $\begin{array}{c}\text { Interpretación } \\
\text { Si aumenta VE en 10\% } \\
\text { entonces el Margen bruto: }\end{array}$ \\
\hline Precio en chacra & 0,663 & Crece $6.63 \%$ & 0,914 & Crece $9.14 \%$ \\
Rendimiento & 0,469 & Crece $4.69 \%$ & 0,330 & Crece $3.30 \%$ \\
Semilla & $-0,184$ & Se reduce 1.84\% & $-0,074$ & Se reduce $0.74 \%$ \\
Pesticidas & $-0,230$ & Se reduce $2.30 \%$ & $-0,043$ & Se reduce $0.43 \%$ \\
Fertilizantes & $-0,213$ & Se reduce $2.13 \%$ & $-0,174$ & Se reduce $1.74 \%$ \\
Mano de obra & $-0,418$ & Se reduce $4.18 \%$ & $-0,110$ & Se reduce $1.10 \%$ \\
Maquinaria & $-0,074$ & Se reduce $0.74 \%$ & $-0,036$ & Se reduce $0.36 \%$ \\
\hline
\end{tabular}

\section{Conflictos de intereses}

Los autores firmantes del presente trabajo de investigación declaran no tener ningún potencial conflicto de interés personal o económico con otras personas u organizaciones que puedan influir indebidamente con el presente manuscrito.

\section{Contribuciones de los autores}

Preparación y ejecución: Pether López; Desarrollo de la metodología: Ramón Diez; Concepción y diseño: Raquel Gómez; Actualización de la literatura: María Anderson; Edición del artículo: Raquel Gómez, Pether López, Ramón Diez, María Anderson.

\section{REFERENCIAS}

- Anderson, M., Gómez, R., Diez, R., \& Linares, A. (2021). Competitividad de Perú y Sudamérica en la producción de arroz (Oryza sativa) y papa (Solanum tuberosum). Anales Científicos, 82(1), 11-21. http://dx.doi.org/10.21704/ac.v82i1.1737

- Aragón, J., Basantes, T., Albuja, L., \& Vásquez, L. (2020). Cadena agroproductiva de la papa (Solanum tuberosum): un análisis en Carchi e Imbabura. Natura@Economía, volumen 5(1),3443. http://dx.doi.org/10.21704/ne.v5i1.1516 
- de Almeida, F. M., Gonçalves Pereira, G. J., Arzuaga Sánchez, J., Torres de la Noval, W., Cabrera Rodríguez, J. A., \& Hernández Jiménez, A. (2015). Principales problemáticas que afectan el desarrollo del cultivo de la papa (Solanum tuberosum L.) en diferentes municipios de la provincia Huambo, Angola. Cultivos Tropicales, 36(4), 100-107. http://scielo.sld.cu/scielo.php?script=sci_arttext\& pid=S0258-59362015000400013

- Basantes, T., Aragón, J., Albuja, L., \& Vásquez, L. (2020). Diagnóstico de los costos, rendimientos de producción y comercialización de papa (Solanum tuberosum L.) en la Zona 1 del Ecuador, año 2019. Revista e-Agronegocios, 6(2). https://doi.org/10.18845/ea.v6i2.5103

- Bonnett, D. (2019). " Resurgencia" y recolonización de la papa. Del mundo andino al escenario alimentario mundial, siglos XVIXX. Anuario Colombiano de Historia Social y de la Cultura, 46(1), 27-57. http://doi.org/10.15446/achsc.v46n1.75552

- Bonnin, R., Cabrera, M., Rivas, H., \& Chamorro, S. (2017). Análisis de la variabilidad espacial del rendimiento del cultivo de soja en una propiedad del Departamento de Distrito Central de Villeta. Investigación Agraria, 19 (1), 16-27. http://scielo.iics.una.py/scielo.php?script=sci_artte xt\&pid=S2305-06832017000100016

- Coaquira-Incacari, R., Julca, A., CoaquiraLastarria, R., \& Mendoza, J. (2019). Caracterización de las unidades productoras de papa (Solanum tuberosum L.) en la provincia de Jauja, Junín, Perú. IDESIA, 37 (4), 101-108. https://www.scielo.cl/pdf/idesia/v37n4/07183429-idesia-37-04-101.pdf

- Figueroa, L., Diez, R., Gómez, R., \& Linares, A. (2019). Beneficios económicos de la semilla certificada en la producción de arroz (Oryza sativa) en Perú. Anales Científicos, 80 (2), 437-451. http://dx.doi.org/10.21704/ac.v80i2.1459

- Guillén, L.\& \& La Rosa, M. (2019). El impacto económico de la regulación ambiental en la producción de papa en Barranca, Lima. Anales científicos, $\quad 80 \quad$ (2), $\quad 409-420$. http://dx.doi.org/10.21704/ac.v80i2.1457

- Lobos, G., Mora, M., Saens, R., Muñoz, T. \& Schnettler, B. (2015). Incluyendo el riesgo en el análisis de viabilidad económica: un modelo de simulación estocástica para las decisiones de inversión de arándanos en Chile. Revista brasileña de fruticultura, $37 \quad$ (4), 870-883. https://www.scielo.br/j/rbf/a/9HDkNL3Zp88cWq gYn JmH4Tz/?lang=en

- López, P. (2019). Rentabilidad y riesgos en la producción de papa blanca comercial. Los casos de Ayacucho y Lima [Tesis de licenciatura, Universidad Nacional Agraria La Molina].

- https://repositorio.lamolina.edu.pe/bitstream/handl e/UNALM/3995/lopez-garciapether.pdf?sequence $=1 \&$ is Allowed $=\mathrm{y}$

- Mejía, G. \& Castellanos, J. (2018). Costos de producción y rentabilidad del cultivo de la papa en Zacapoaxtla, Puebla. Revista Mexicana de Ciencias Agrícolas, volumen 9 (8). https://doi.org/10.29312/remexca.v9i8.1721

- Minaya, C. (2015). Análisis de la rentabilidad en la producción de papas blancas comerciales en las regiones de Huánuco y Lima. Anales científicos 76 (2), 369-375. http://dx.doi.org/10.21704/ac.v76i2.803

- Oliver, J. (2017). Rendimiento de dos variables de papa (Solanum tuberosum L.) con la aplicación de tierra negra y fertilizantes inorgánicos. Revista de Investigación e Innovación Agropecuaria y de Recursos Naturales, 4(2), 5669.http://www.scielo.org.bo/scielo.php?script=sci _arttext\&pid=S2409-16182017000200008

- Scott, G. (1985). Mercados, mitos e intermediarios. Un estudio de la comercialización de la papa en la zona central del Perú (pp. 308). Centro de Investigación de la Universidad del Pacífico (CIUP). p.308.

file://C:/Users/Usuario/Downloads/MercadosMit oseIntermediarios\%20(2).pdf 